\title{
Benchmark of the SLACCAD code against data from the MANITU testbed at IPP
}

\author{
P. Agostinetti ${ }^{\mathrm{a}}$, G. Chitarin ${ }^{\mathrm{a}, \mathrm{b}}$, P. Franzen ${ }^{\mathrm{c}}$, B. Ruf ${ }^{\mathrm{c}}$, G. Serianni ${ }^{\mathrm{a}}$ and P. \\ Veltri ${ }^{\mathrm{a}}$ \\ ${ }^{a}$ Consorzio RFX, Euratom-ENEA Association, C.so Stati Uniti 4, I-35127, Padova, Italy \\ ${ }^{b}$ Dept. of Engineering and Management, University of Padova, Vicenza, Italy \\ ${ }^{c}$ Max-Planck-Institut für Plasmaphysik, Boltzmannstr. 2, D-85748 Garching, Germany
}

\begin{abstract}
.
A set of physics and engineering codes is currently used and developed at Consorzio RFX to simulate the negative ion beam accelerators of the next experiments SPIDER and MITICA. In order to verify the accuracy of the simulations, an experimental validation has been undertaken, that consist in benchmarking the codes with data from ITER-relevant experiments. As a first step, the SLACCAD code have been applied to simulate the beam optics in the MANITU testbed at IPP, which features a Radio Frequency ion source and is able to operate with ITER-like conditions in terms of extracted ion current density and pulse length. A description of the modeling activities and a discussion of the main results obtained so far are here reported. In particular, the effect of beam halo, space charge compensation and particle angle at the emitter are investigated. Some results will be used to identify reasonable operating parameters as inputs for the design optimization of the SPIDER and MITICA experiments.
\end{abstract}

Keywords: Neutral Beam Injector, beam, modeling

PACS: $41.75 . \mathrm{Cn}, 52.50 . \mathrm{Gj}$

\section{INTRODUCTION}

The Radio Frequency (RF) driven ion source developed at IPP Garching (Germany), sketched in Fig. 1a, has been chosen by the ITER board as the reference source for the ITER Neutral Beam Injector (NBI) system due to the convenience of its maintenancefree operation and to the recent improvement of the RF source performances in terms of current density and reliability [1, 2, 3, 4, 5]. The long pulse stability has been demonstrated at the test facility MANITU, able to routinely operate ITER relevant parameters.

In the framework of the support activities for the ITER NBIs, the PRIMA test facility, which includes a RF-driven source with $100 \mathrm{keV}$ accelerator (SPIDER) and a complete $1 \mathrm{MeV}$ Neutral Beam system (MITICA), is under construction at Consorzio RFX in Padova [6, 7].

A comprehensive set of codes is currently being used and developed at Consorzio RFX, aiming at simulating the most important phenomena inside the negative ion electrostatic accelerator: electrical and magnetic fields, beam aiming and optics, pressure and density of the background gas, stripping reactions, transmitted and dumped power, stresses and deformations of the accelerator grids. These codes are necessary for the engineering design optimization of most components of the injector system (ion source, 
(a)

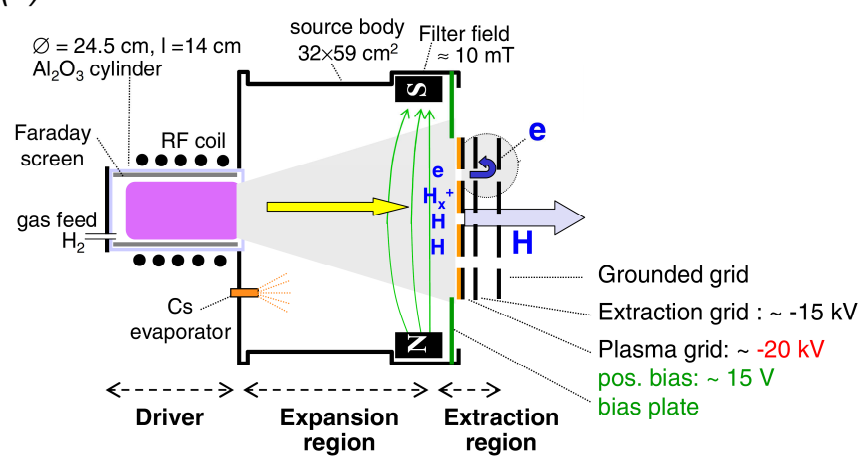

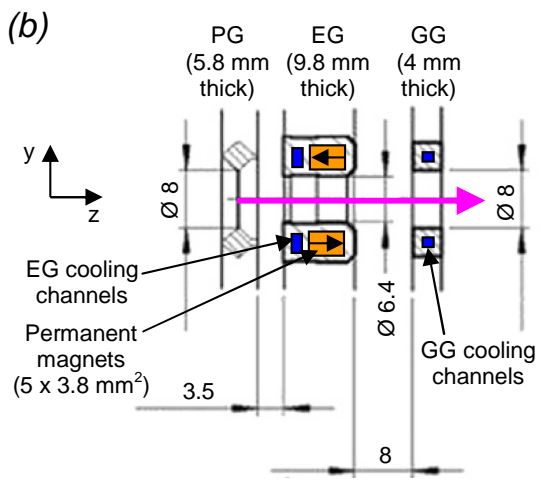

FIGURE 1. IPP RF-driven ion source: (a) Overall scheme; (b) geometry of the grids in the MANITU experiment.

accelerator, neutralizer, electron and ion dumps, calorimeter) as well as for the prediction of the operating conditions and the interpretation of the experimental results.

The application of the SLACCAD single beamlet optics code to the MANITU experiment is described here. The results of simulations are compared and discussed in relation with the experimental results.

The main goal of this comparison is to answer the following questions:

1. Is the optimal perveance condition properly evaluated using the SLACCAD code? The optimal perveance is defined as the operating condition where the beamlet divergence reaches its minimum. It represents the ideal condition for the operation of neutral beam injectors in fusion experiments.

2. What is a reasonable fraction of beam current to be assumed as beam halo? For negative ion beams, the most considered hypothesis on halo formation (see for example [8]) is that it could be generated on the downstream side of the Plasma Grid (PG), where there could be suitable conditions for the surface generation of $\mathrm{H}^{-} / \mathrm{D}^{-}$(in terms of grid temperature, cesium coverage and presence of $\mathrm{H}^{0} / \mathrm{D}^{0}$ atoms that can impinge on the surface with proper energy). In the current design of the MITICA accelerator [9, 10], halo particles are foreseen to contribute for a significant percentage of the total power impinging on the grids (about one third). Hence, it is very important, in particular for the design of the MITICA accelerator and related cooling systems, to assume a reasonable halo current. The only evaluations available so far in ITER-relevant experiments are the ones made with an infrared camera and a carbon beam target at the SINGAP experiment in CEA-Cadarache (France), where an $8 \%$ halo was measured [8]. However, this evaluation might be affected by the fact that the SINGAP ion source was a filamentarc chamber, whilst a RF-driven ion source is foreseen in SPIDER, MITICA and ITER HNB.

3. What is the role and size of the Space Charge Region (SCR) located downstream of the accelerator? Space charge has an influence on beam optics, as it makes particles repel each other. This effect acts inside the accelerator, where the positive particles generated by stripping reactions are drained away by the electric field. On 
the other hand, it is not present far downstream of the accelerator, because, due to the absence of electric field, the positive particles remain inside the beam, giving an attractive force that compensates for the space charge due to the negative ions. From plasma theory $[11,12]$, it appears that space charge should be considered also in a small region located just downstream of the accelerator exit, where space charge compensation builds up. The length of this region (see SCR in Fig. 2a), is foreseen to be in the range of a few centimeters, depending on the accelerator parameters (particle type, energy, pressure etc.).

4. Does the initial angle of the particles assumed at the beam emitter have an influence on the calculated beam divergence? The negative ions $\left(\mathrm{H}^{-}\right.$or $\left.\mathrm{D}^{-}\right)$are generated by surface reactions on the upstream surface of the PG and then they are deflected toward the apertures. Hence, they could reach the aperture meniscus with a nonaxial direction. The assumptions on the initial angle between the particle velocity and the beamlet axis could have an influence on the beam divergence.

\section{EXPERIMENTAL DATA}

The MANITU testbed at IPP features a Radio Frequency ion source, able to operate with long pulses (up to one hour) both in hydrogen and in deuterium. The extraction/acceleration system, sketched in Fig. 1b, is made of a Plasma Grid (PG), an Extraction Grid (EG) and a Grounded Grid (GG).

A pertinent quantity for comparing data from hydrogen and deuterium pulses (as well as data from different ion sources) is the ratio $P / P_{0}$ (also called normalized perveance) between perveance $P$ and theoretical perveance of a planar diode $P_{0}$.

Perveance describes the beam extraction from a plasma, indicating how significant the space charge effect is on the beam's motion. For the extraction system of an ion source, it can be calculated as the coefficient of proportionality between the space-charge limited extracted current $I_{e x t}$, and the extraction voltage $V_{e x t}$ to the three-half power:

$$
P=\frac{I_{e x t}}{V_{e x t}^{\frac{3}{2}}}
$$

Using the Child-Langmuir law, the theoretical perveance of a planar diode $P_{0}$ can be written as:

$$
P_{0}=\frac{4 \pi \varepsilon_{0}}{9} \sqrt{\frac{2 e}{m}}\left(\frac{r}{d}\right)^{2}
$$

where $\varepsilon_{0}$ is the vacuum dielectric constant, $e$ the electron charge, $m$ the mass of the extracted ions, $r$ the PG aperture radius, and $d$ the distance between the PG and EG.

Tab. 1 reports some recent experimental data of MANITU, recorded during hydrogen and deuterium campaigns and setting different potentials for the grids. The beam divergence $\theta_{D S S}$ have been measured by means of Doppler Shift Spectroscopy, with the procedure described in [13]. The voltages $V_{e x t}$ and $V_{t o t}$ and the extracted ion current density $j_{\text {ext }}$ have been measured on the electric power supplies. 
TABLE 1. Experimental data measured during different pulses in MANITU using hydrogen and deuterium ions. $j_{e x t}$ is the extracted ion current density. $V_{e x t}$ is the extraction voltage, equal to $V_{E G}-V_{P G}$, while $V_{t o t}$ is the total voltage, equal to $V_{G G}-V_{P G} . P / P_{0}$ is the normalized perveance, where $P$ is the perveance $\left(=I_{e x t} / V_{e x t}^{3 / 2}\right)$ and $P_{0}$ is the theoretical perveance of a planar diode. $\theta_{D S S}$ is the average divergence angle measured by Doppler Shift Spectroscopy (DSS) with the procedure described in [13].

\begin{tabular}{ccccccc}
\hline Pulse & Isotope & $j_{\text {ext }}\left[A m^{-2}\right]$ & $V_{\text {ext }}[k V]$ & $V_{\text {tot }}[k V]$ & $P / P_{0}$ & $\theta_{D S S}[$ degrees $]$ \\
\hline 86486 & $\mathrm{H}^{-}$ & 112.5 & 3.7 & 24.3 & 0.116 & 2.71 \\
86487 & $\mathrm{H}^{-}$ & 138.7 & 4.6 & 25.2 & 0.102 & 2.40 \\
86488 & $\mathrm{H}^{-}$ & 158.2 & 5.6 & 26.2 & 0.086 & 2.47 \\
86489 & $\mathrm{H}^{-}$ & 171.6 & 6.6 & 27.2 & 0.073 & 2.75 \\
86490 & $\mathrm{H}^{-}$ & 182.7 & 7.6 & 28.2 & 0.063 & 3.04 \\
86491 & $\mathrm{H}^{-}$ & 186.3 & 7.9 & 28.5 & 0.060 & 3.07 \\
\hline 87850 & $\mathrm{D}^{-}$ & 44.0 & 2.5 & 18.0 & 0.121 & 2.17 \\
87851 & $\mathrm{D}^{-}$ & 68.0 & 3.5 & 18.9 & 0.113 & 1.89 \\
87852 & $\mathrm{D}^{-}$ & 94.6 & 4.5 & 19.9 & 0.106 & 2.17 \\
87853 & $\mathrm{D}^{-}$ & 111.8 & 5.5 & 20.9 & 0.091 & 2.82 \\
87854 & $\mathrm{D}^{-}$ & 117.9 & 6.0 & 21.4 & 0.084 & 3.12 \\
87855 & $\mathrm{D}^{-}$ & 121.6 & 6.5 & 21.9 & 0.077 & 3.45 \\
87856 & $\mathrm{D}^{-}$ & 124.8 & 7.0 & 22.4 & 0.071 & 3.53 \\
87857 & $\mathrm{D}^{-}$ & 129.9 & 7.5 & 22.9 & 0.067 & 3.75 \\
87858 & $\mathrm{D}^{-}$ & 130.7 & 7.9 & 23.3 & 0.062 & 3.87 \\
87859 & $\mathrm{D}^{-}$ & 133.7 & 8.5 & 23.9 & 0.057 & 3.9 \\
87860 & $\mathrm{D}^{-}$ & 136.2 & 9.0 & 24.5 & 0.053 & 4.05 \\
\hline
\end{tabular}

\section{BEAM MODELING}

The SLACCAD code has been used to estimate the electric field inside the accelerator by integration of the Poisson's equation, with cylindrical geometry conditions [14]. This is a modified version of the SLAC Electron Trajectory Program [15], adapted to include ions, a free plasma boundary and a stripping loss module [16]. Stripping losses were measured to be quite low in MANITU, around 2-3\% [13]. Hence, the decrease of space charge along the accelerator due to the stripping losses is expected to be very small and is here neglected.

A typical output of the SLACCAD code is shown in Fig. 2. The grids act as electrostatic lenses and the beamlet meniscus corresponds to the first equipotential line $(0 \mathrm{kV})$. Beam core and beam halo are simulated at the same time, with the particles constituting the beam halo starting from the downstream side of the PG. The core has a lower divergence angle (in the range between 0.5 and 3 degrees, depending on normalized perveance); in fact, the beam core at the exit plane appears to be nearly cylindrical at the Target Plane (TP). On the other hand, beam halo has a higher divergence (in the range between 6 and 10 degrees) and appears like a diverging conus.

A suitable parameter for the evaluation of single beam optics with SLACCAD is the average divergence $\theta_{S L}$ at the exit plane. This is calculated as a weighted root mean square of the single ray divergence $\theta_{i}$ at the TP, taking as weight the current $\sigma_{i}$ carried 
(a)

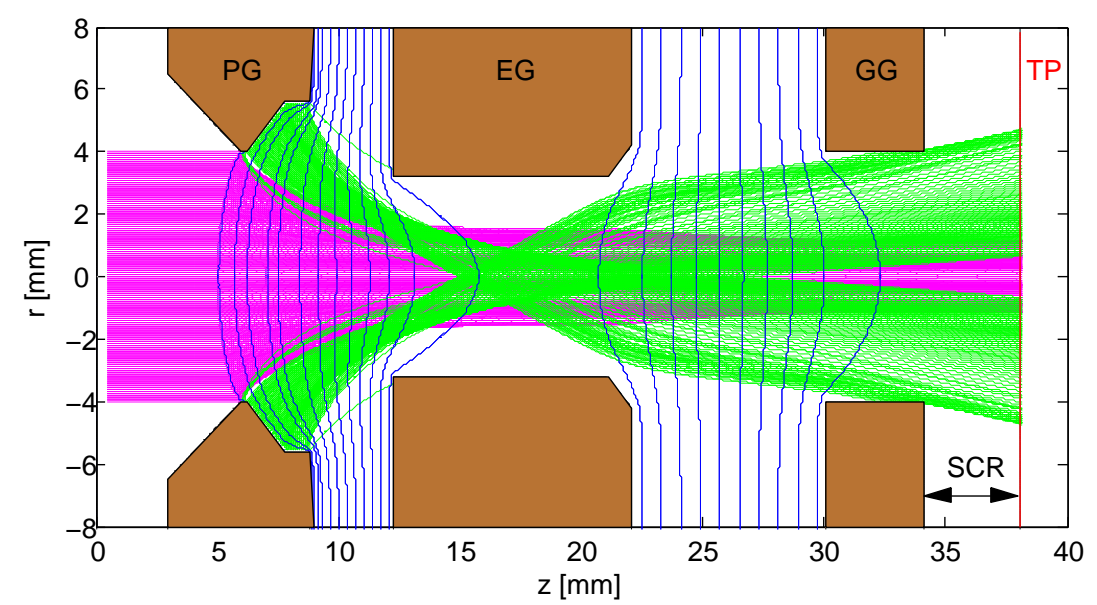

(b)

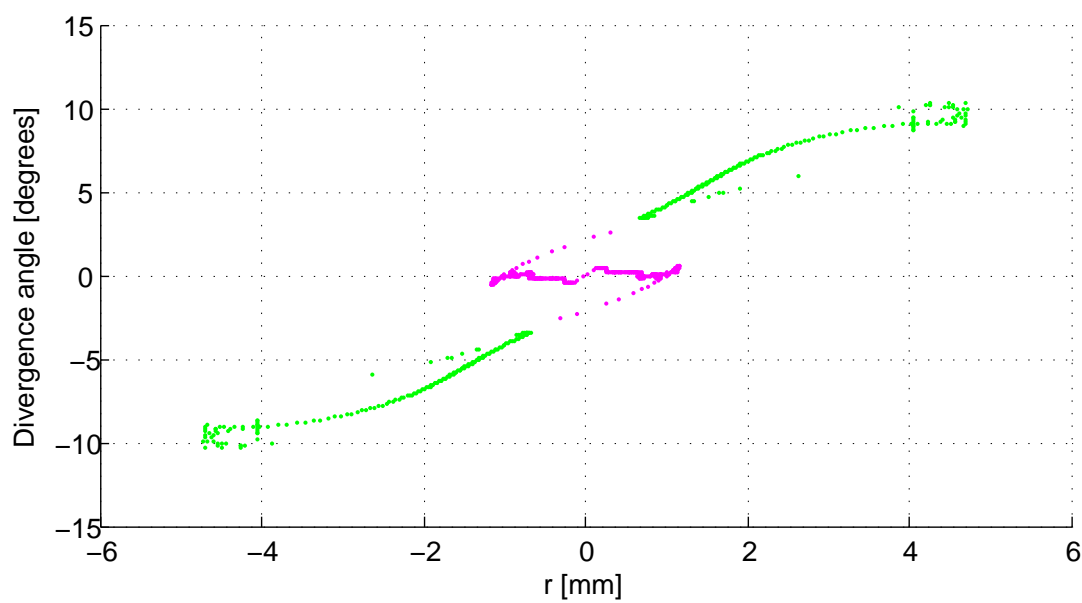

FIGURE 2. Main outputs of SLACCAD code: (a) Trajectories and equipotential lines; (b) Emittance plot at the TP. Core and halo particles are plotted in magenta and green, respectively. The boundary conditions of this analysis are: pulse 86487 (hydrogen), emitter $1, I_{\text {halo }} / I_{\text {core }}=12 \%$ and SCR=4 $\mathrm{mm}$.

by each ray, according to the formula:

$$
\theta_{S L}=\sqrt{\frac{\sum \sigma_{i} \theta_{i}^{2}}{\sum \sigma_{i}}}
$$

Only the particles reaching the TP are taken into account for the calculation of $\theta_{S L}$, while the halo particles impinging on the GG are excluded. In this way, $\theta_{S L}$ can be compared with $\theta_{D S S}$, that is measured 1.2-1.5 m downstream of the GG [1].

A sensitivity analysis has been carried out to investigate the variation of $\theta_{S L}$ with the following parameters:

1. Emitter type. Four different emitters (shown on the left part of Fig. 5) are considered. Emitter 1 is the one currently considered for the design of SPIDER and 

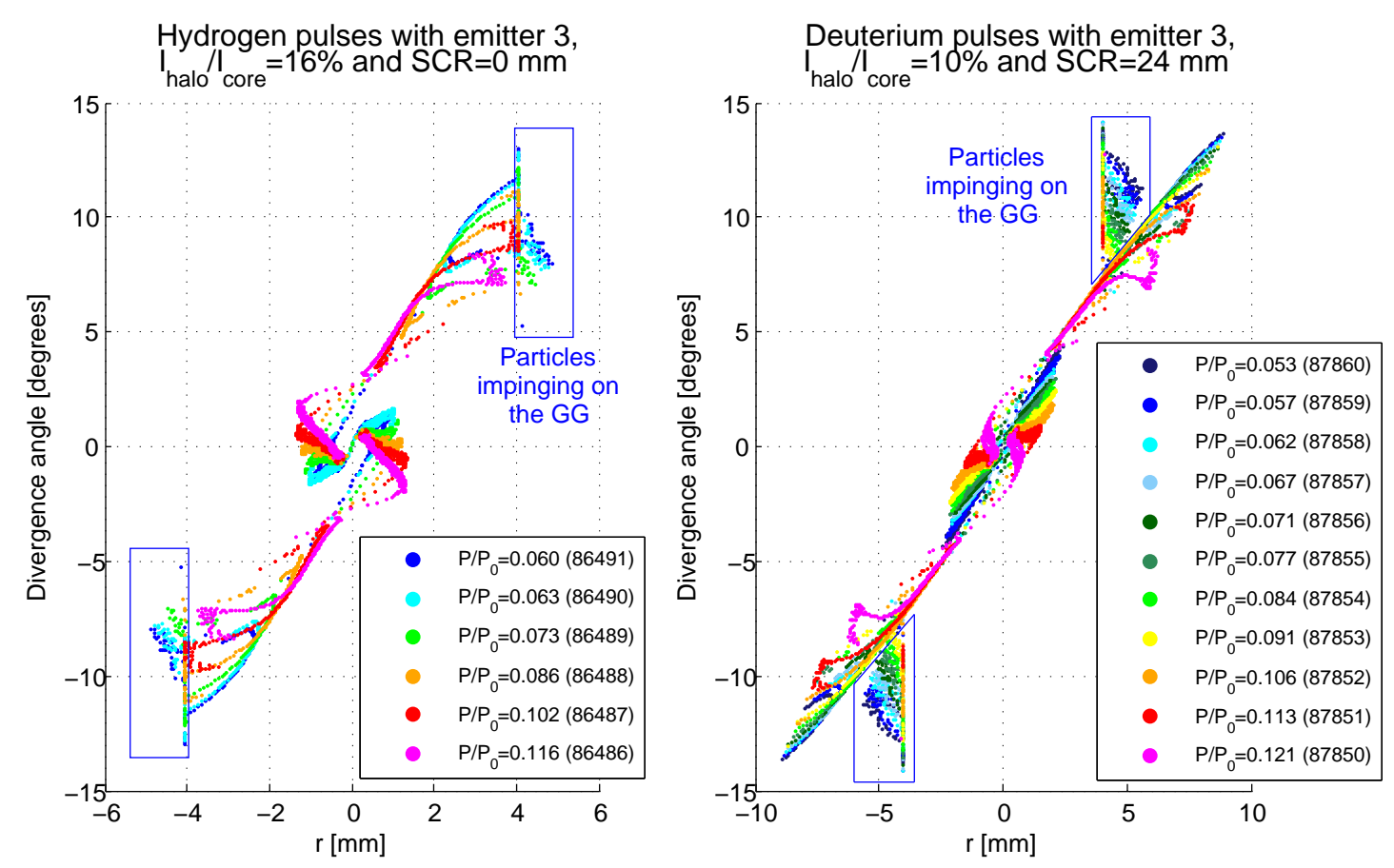

FIGURE 3. Emittance plots of the single beamlet for all the pulses. The best fitting conditions are here considered. The particles impinging on the GG are highlighted; these particles do not reach the TP and for this reason are not considered in $\theta_{S L}$ calculations.

MITICA; it has $8 \mathrm{~mm}$ diameter (as the PG aperture) and axial velocity for all particles. Emitter 2 is like emitter 1 but with a smaller diameter $(7.5 \mathrm{~mm}$ instead of 8 $\mathrm{mm})$. Emitter 3 takes into account a possible inclination of the initial particles with respect to the aperture axis; in fact, one third of the particles have an axial direction, one third are bent of $10^{\circ}$ toward the beamlet axis and one third $10^{\circ}$ away from the beamlet axis. Emitter 4 is like emitter 3 but with $\pm 20^{\circ}$ angles.

2. Halo current. The simulations are made considering an halo current ranging between 0 and $18 \%$ of the core current. In any case, halo particles are starting from the downstream surface of the PG aperture (see Fig. 2a).

3. Length of the SCR between the GG exit and the target plane (TP). This is varied in the range $0-44 \mathrm{~mm}$.

Fig. 3 shows an example of emittance plots of the single beamlet for all the pulses. The best fitting conditions (identified in the following) are considered: emitter 3, $I_{\text {halo }} / I_{\text {core }}=16 \%$ and SCR=0 $\mathrm{mm}$ for hydrogen pulses; emitter $3, I_{\text {halo }} / I_{\text {core }}=10 \%$ and $\mathrm{SCR}=24 \mathrm{~mm}$ for deuterium pulses. A minimum divergence condition is obtained when the beamlet is approximately cylindrical (not convergent nor divergent); this corresponds to an almost flat emittance plot of the core particles. Pulses 86488 (for hydrogen) and 87851 (for deuterium) appear to be the closest to this condition. 

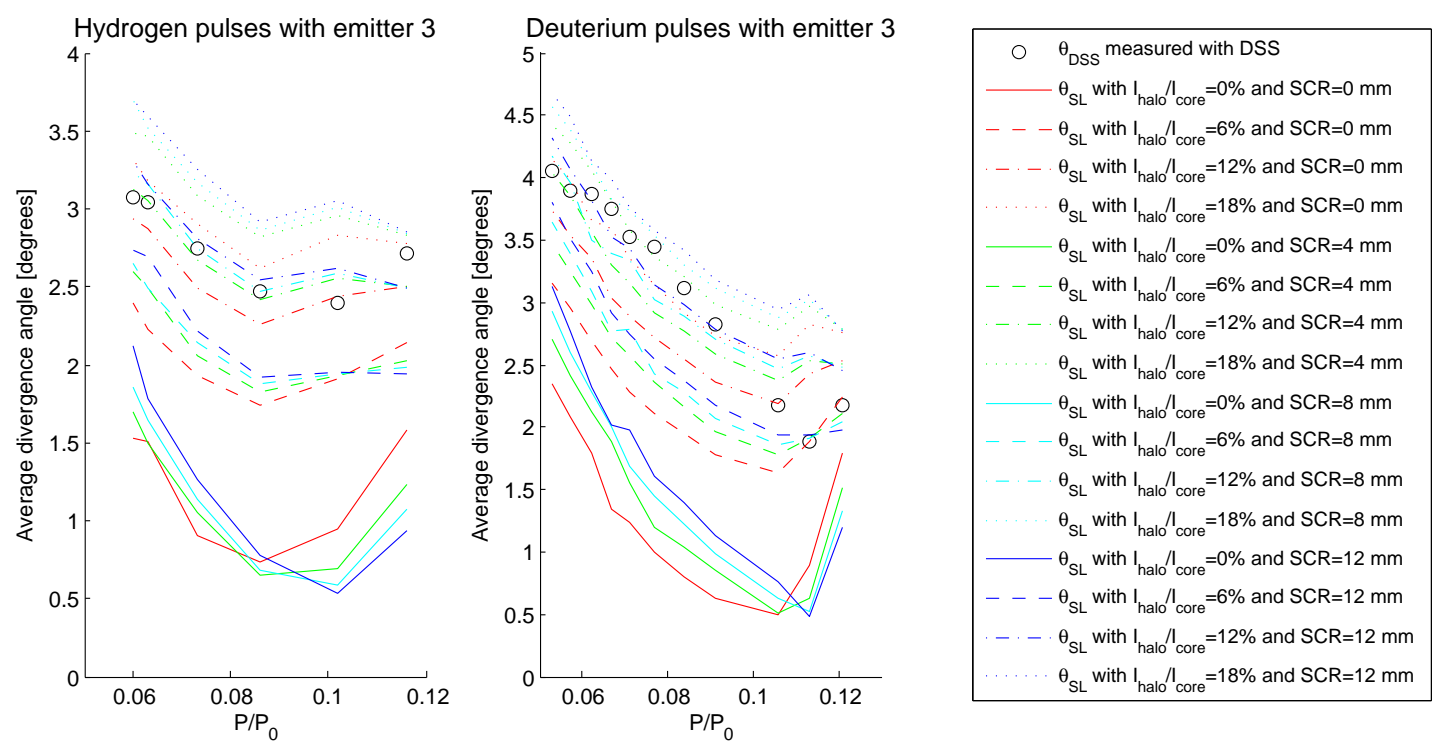

FIGURE 4. Simulated and measured beam divergence. Emitter 3 is here considered, with four values of halo current $(0,6,12$ and $18 \%$ of core current) and four values of SCR $(0,4,8$ and $12 \mathrm{~mm})$.

\section{COMPARISON BETWEEN MEASUREMENTS AND SIMULATIONS}

A large number of simulations have been carried out assuming different hypotheses for emitter, halo and SCR.

Fig. 4 shows the measured (dots) and calculated (curves) values of the average divergence angles as a function of the normalized perveance $P / P_{0}$. In these plots, the results with emitter 3 , four values of halo current $(0,6,12$ and $18 \%$ of core current) and four values of $\operatorname{SCR}(0,4,8$ and $12 \mathrm{~mm})$ are considered. It can be observed that:

- The relative perveance value $P / P_{0}$ corresponding to the minimum divergence obtained from the simulations is always consistent with the value obtained from the measurements. In fact, the minimum points of the simulation curves (in the range $[0.086 ; 0.102]$ for $\mathrm{H}^{-}$and $[0.106 ; 0.113]$ for $\mathrm{D}^{-}$) are generally close to the lowest measured points $\left(0.102\right.$ for $\mathrm{H}^{-}$and 0.113 for $\left.\mathrm{D}^{-}\right)$. On the other hand, if halo is not considered, the calculated divergence values $\theta_{S L}$ is significantly lower than the measured values $\theta_{D S S}$. A similar result (with a good estimation of the optimal perveance but an underestimation of the divergence angles) was found by comparing the MANITU data with the results of the KOBRA code [1]. In that case, the larger divergence calculated by the code was explained by the authors with the effect of the space charge.

- The curves shift upward with increasing halo current; in fact, halo divergence is much higher than core divergence.

- Without halo (continuous curves), the curves tend to shift rightward with increasing values of SCR; this is probably due to the fact that the space charge effect increases 
Hydrogen pulses
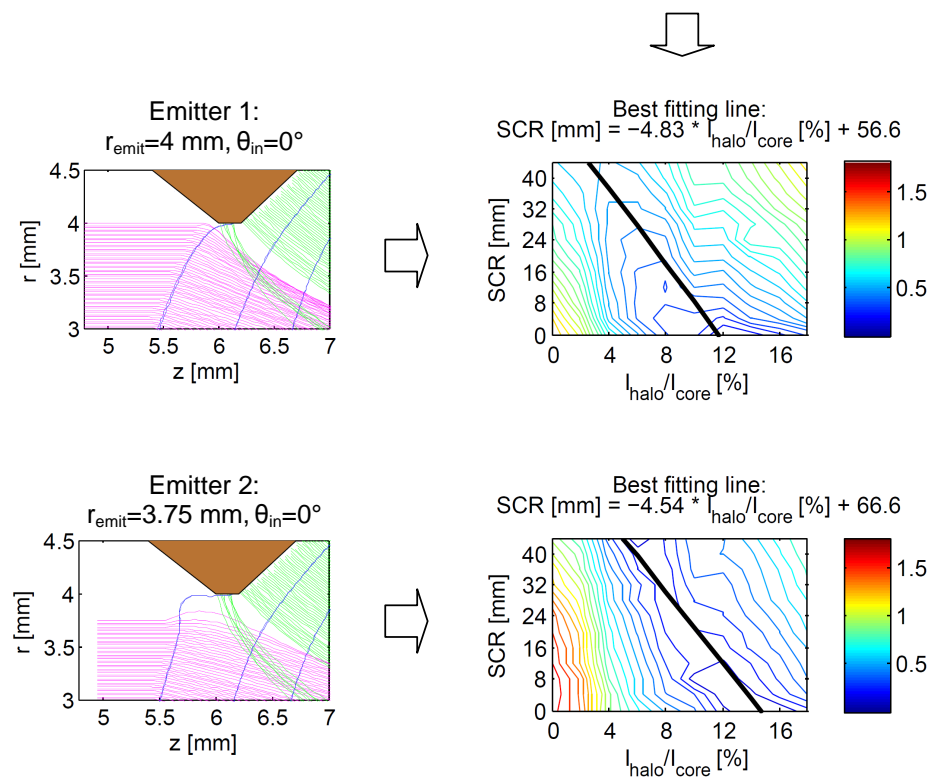

Emitter 3:
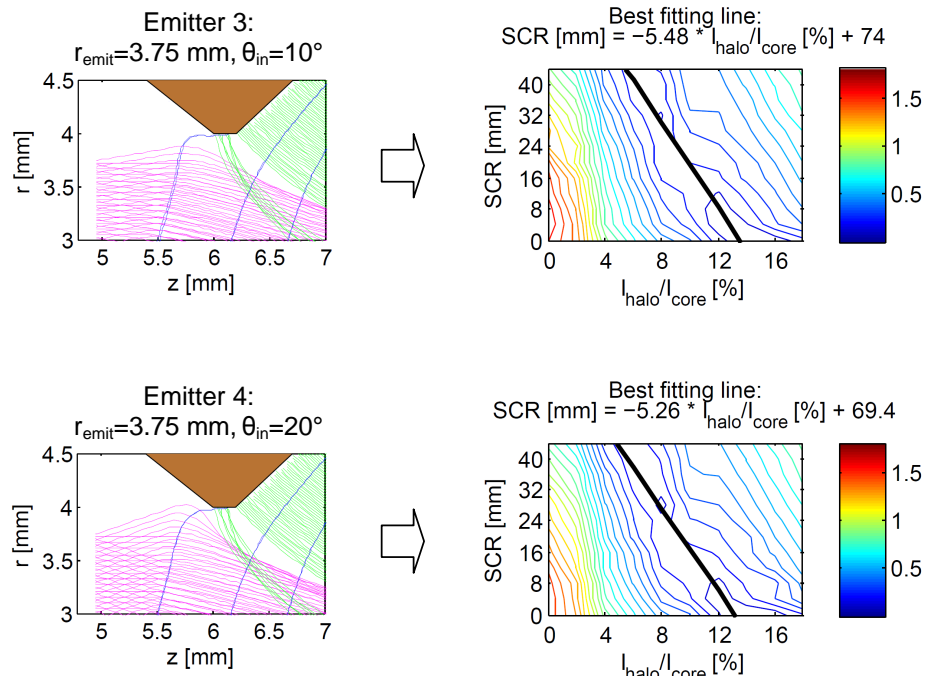
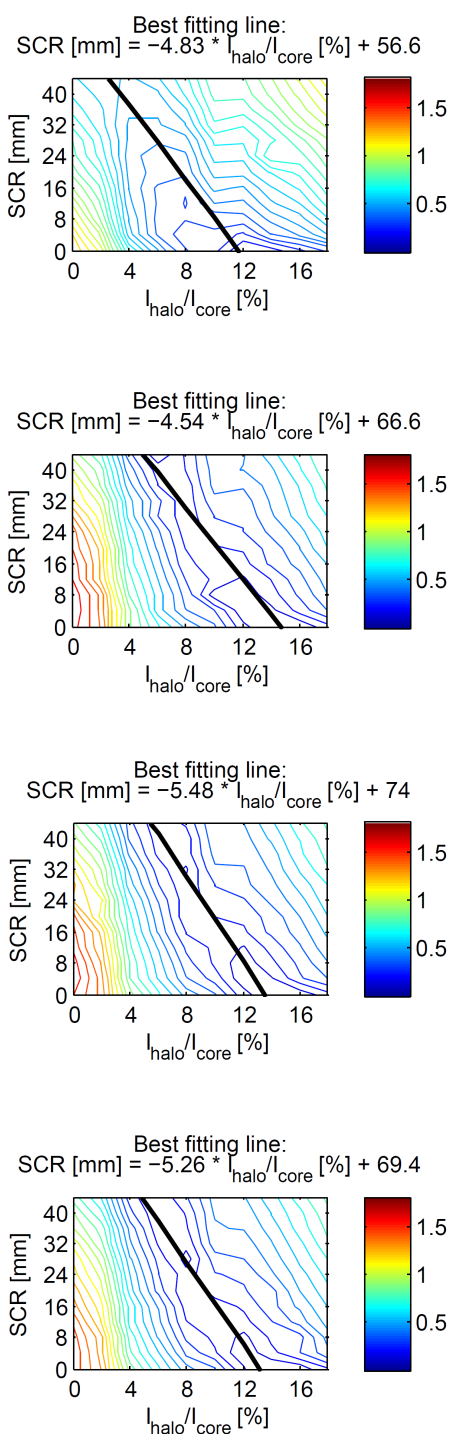
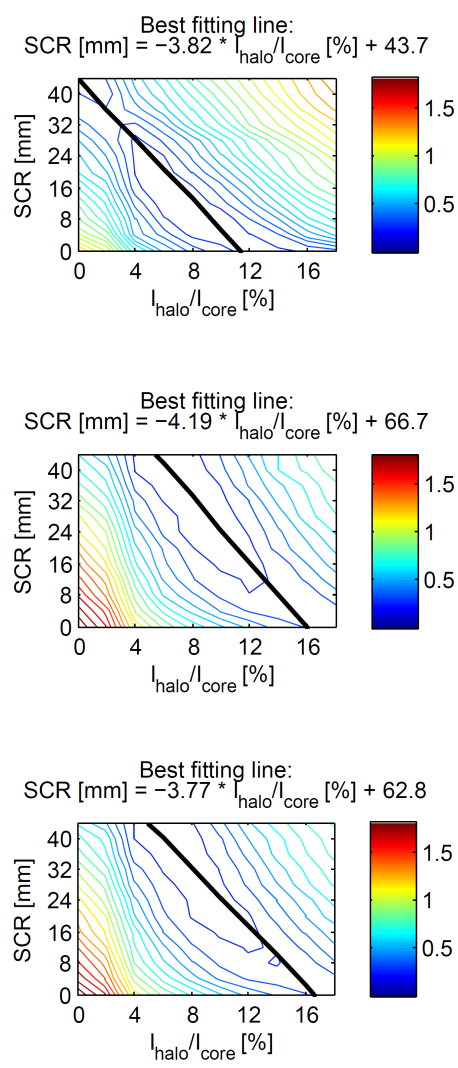

Deuterium pulses
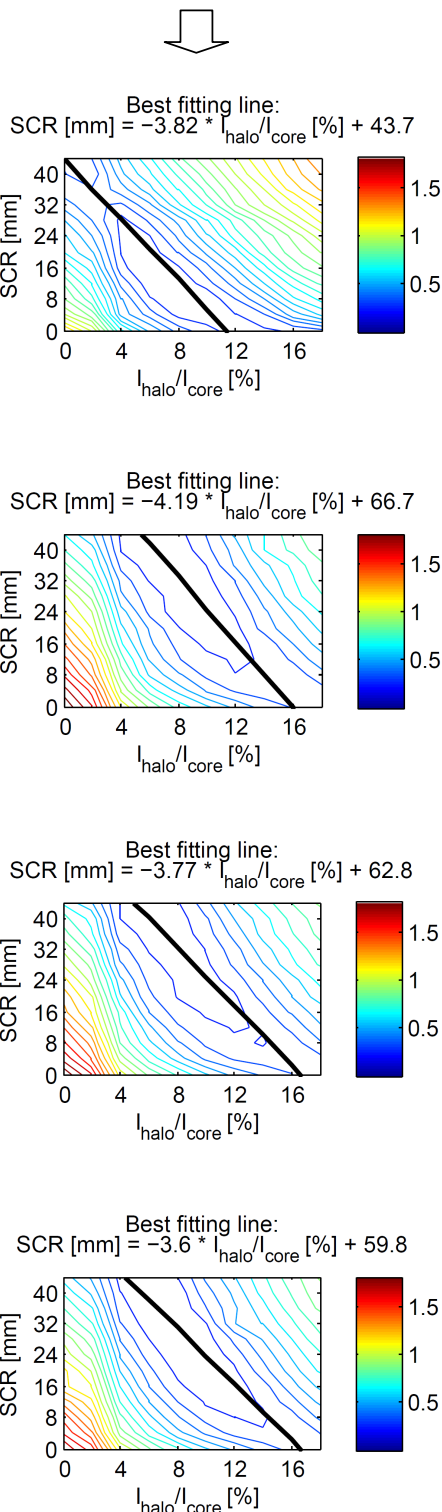

FIGURE 5. Fitting of experimental data using different simulation assumptions. The divergence fitting error $\Delta \theta_{\text {avg }}$ is plotted as a function of halo current (from 0 to $18 \%$ of the core current) and length of SCR (from 0 to $44 \mathrm{~mm}$ ) for different emitter types (1, 2, 3 or 4) and gas types $\left(\mathrm{H}^{-}\right.$or $\mathrm{D}^{-}$). For each case, the best fitting line is plotted and the corresponding equation is reported above the plot, representing the most reasonable combinations of halo current and SCR.

the beam core divergence and hence moves the optimal condition (minimum divergence) to higher values of perveance.

- In the cases with halo (all curves except the continuous ones), the curves tend to shift upward with increasing values of SCR. In this case, the effect of space charge on beam halo is probably larger than the one on beam core; hence, the halo 
divergence increases and also the overall divergence. It can be observed that this effect is similar the one given by an increase of halo percentage.

- The curves tend to be more flat on the right part of the plots (higher $P / P_{0}$ ) with increasing halo percentage; the reason for this effect could be that a large fraction of halo particles impinge on the GG and does not reach the TP.

In order to select the most realistic simulation assumptions and the best beam divergence fitting line, the fitting error $\Delta \theta_{\text {avg }}$ has been calculated for all cases, defined as the average difference between the simulated divergence $\theta_{S L}$ and the experimental data $\theta_{D S S}$. For example, considering the left plot (hydrogen pulses) of Fig. 4, the $\Delta \theta_{\text {avg }}$ of the case with $I_{\text {halo }} / I_{\text {core }}=0 \%$ and SCR=0 $\mathrm{mm}$ has been calculated as the average distance between the red continuous curve and the dots.

The $\Delta \theta_{\text {avg }}$ values, calculated in any combination of the four emitters, ten values of halo current $(0,2,4,6,8,10,12,14,16$ and $18 \%$ of core current) and twelve values of SCR length $(0,4,8,12,16,20,24,28,32,36,40$ and $44 \mathrm{~mm})$, are summarized in Fig. 5. It can be observed that the best fitting areas (regions with the lowest values of $\left.\Delta \theta_{\text {avg }}\right)$ have the shape of diagonal strips on the $\left(I_{\text {halo }} / I_{\text {core }}\right) /$ SCR plots. Best fitting lines have been calculated for each case with an automatic fitting tool. The corresponding equations are reported over each graph, representing the most reasonable combinations of halo current and SCR. As SCR can be estimated with plasma theory, halo current can be then evaluated with the equations of the best fitting lines in Fig. 5.

As an example, if we consider SCR equal to $30 \mathrm{~mm}$, the corresponding values of $I_{\text {halo }} / I_{\text {core }}$ (considering emitter 1,2,3 and 4, respectively) are:

- for hydrogen pulses: $5.5,8.1,8,7.5 \%$

- for deuterium pulses: $3.6,8.7,8.7,8.3 \%$

Finally, both for hydrogen and deuterium pulses, the lowest values of $\Delta \theta_{\text {avg }}$ are reached with emitter 3 ; hence, this can be considered to be the most realistic emitter among the four considered ones.

\section{CONCLUSIONS}

Experiments carried out on the MANITU testbed at IPP Garching have been simulated using the SLACCAD code. The simulation results have been compared against the experimental data, in particular against those obtained with the Doppler Shift Spectroscopy (DSS) diagnostic system.

The main results are:

- The estimations of the optimal perveance corresponding to minimum beam divergence are in good agreement with the experiments both with hydrogen and deuterium pulses.

- A relationship has been found between the halo current and the space charge region.

- The most realistic emitter is \#3, featuring a $\pm 10^{\circ}$ initial angle of the particles. 
Possible next steps are:

- The density inside the accelerator could be estimated with dedicated codes.

- Stripping losses and heat loads could be calculated with the EAMCC code [17]. Then, the former could be compared with DSS measurements (with the procedure described in [13]), and the latter with calorimetric measurements.

- More pulses could be considered to obtain better statistics.

- Other parameters could be varied in SLACCAD, together with emitter, halo percentage and SCR.

\section{ACKNOWLEDGEMENTS}

This work was set up in collaboration and financial support of F4E. The authors would like to thank Dr. H.P.L. de Esch for the support and the fruitful discussions.

\section{REFERENCES}

1. U. Fantz, et al., Physical performance analysis and progress of the development of the negative ion RF source for the ITER NBI system, Nucl. Fusion 49 (2009) 125007.

2. A. Stäbler, et al., Development of a RF-driven ion source for the ITER NBI system, Fus. Eng. and Design 84 (2009) 265-268.

3. P. Franzen, et al., Physical and experimental background of the design of the ELISE test facility, AIP Conf. Proc. 1097 (2009) 451.

4. W. Kraus, et al., Long pulse $\mathrm{H}^{-}$beam extraction with a rf driven ion source on a high power level, Rev. Sci. Instrum. 81, 02B110 (2010).

5. U. Fantz, et al., Spectroscopy - a powerful diagnostic tool in source development, Nucl. Fusion 46 (2006) S297-S306.

6. P. Agostinetti, et al., Physics and engineering design of the Accelerator and Electron Dump for SPIDER, Nucl. Fusion 51 (2011) 063004.

7. P. Sonato, et al., The ITER full size plasma source device design, Fusion Eng. Des. 84 (2009), 269-274.

8. H.P.L. de Esch, L. Svensson, Negative ion beam halo mitigation at the 1MV testbed at IRFM, Fusion Eng. Des. 86 (2011) 363-368.

9. P. Zaccaria, et al., Progress in the MITICA beam source design, Rev. Sci. Instrum. 83, $02 B 108$ (2012).

10. G. Chitarin, et al., Concepts for the magnetic design of the MITICA neutral beam test facility ion accelerator, Rev. Sci. Instrum. 83, 02B107 (2012).

11. P. Veltri, et al., Study of space charge compensation phenomena in charged particle beams, Rev. Sci. Instrum. 83, 02B709 (2012).

12. P. Spädtke and R. Hollinger, Beam transport, in The Physics and Technology of Ion Sources, edited by I. G. Brown (Wiley, New York, 1989).

13. P. Franzen, et al., Beam Homogeneity Dependence on the Magnetic Filter Field at the IPP Test Facility MANITU, AIP Conference Proceedings, Vol. 1390 (2011).

14. J. Pamela, A model for negative ion extraction and comparison of negative ion optics calculations to experimental results. Rev. Sci. Inst. 62 (1991), 1163-1172.

15. W.B. Hermannsfeld, Electron Trajectory Program, SLAC report, Stanford Linear Accelerator Center, SLAC-226 (1979).

16. H.P.L. de Esch, R.S. Hemsworth and P. Massmann. Updated physics design ITER-SINGAP accelerator. Fusion Eng. Des. 73 (2005) 329-341.

17. G. Fubiani et al., Modeling of secondary emission processes in the negative ion based electrostatic accelerator of the International Thermonuclear Experimental Reactor. Phys. Rev. Special Topics Accelerators and Beams 11, 014202 (2008). 